\title{
Genetic Diversity and Relationship of China's Bergenia Germplasm Revealed by Intersimple Sequence Repeat Markers
}

\author{
Xiuli Lv \\ Shanghai Academy of Landscape Architecture Science and Planning, and Shanghai Engineering \\ Research Center of Landscaping on Challenging Urban Sites, Shanghai 200232, China \\ Yuan Guan \\ Forestry and Fruit Tree Research Institute, Shanghai Academy of Agricultural Sciences, \\ Shanghai 201403, China \\ Jian Wang \\ Yanwei Zhou \\ Jiangsu Key Laboratory for the Research and Utilization of Plant Resources, Jiangsu Province \\ and Chinese Academy of Sciences, Nanjing 210014, China
}

Qunlu Liu

School of Design, Shanghai Jiao Tong University, Shanghai 200240, China

\author{
Zequn Yu \\ Shanghai Gardening-Landscaping Construction Co., Ltd., Shanghai 200333, China
}

AdDitional INDEX wORDs. breeding parents, cluster analysis, germplasm selection, ISSR

\begin{abstract}
To reveal the genetic diversity and genetic relationships of China's Bergenia germplasm, 28 Bergenia accessions from different regions in China were analyzed by 24 intersimple sequence repeat (ISSR) markers. The results showed that 318 sites were amplified in all germplasm, including 307 polymorphic sites, and the percentage of polymorphic sites was $\mathbf{9 6 . 5 4 \%}$. Cluster analysis showed that the 28 accessions were divided into three categories, with a similarity coefficient of $\mathbf{0 . 5 4 7 5}$. Bergenia purpurascens was clustered into one category; $B$. scopulosa was clustered into one category; and B. tianquaninsis, B. emeiensis, B. stracheyi, and B. crassifolia were clustered into one category. The results of the cluster analysis indicated that the 28 accessions were not completely classified by origin. Using the ISSR marker technique to analyze the phylogenetic relationship of Bergenia germplasm is helpful for identifying valuable resources and providing a theoretical basis for the selection of breeding parents.
\end{abstract}

Bergenia is a perennial herb, a genus included in the family of Saxifragaceae. It is a wildflower with beautiful foliage, distributed in high-altitude areas across China, and can be used as a Chinese herbal medicine (Wang et al., 2006). Studies on diversity within Bergenia have been mostly limited to $B$. purpurascens in a narrow region of China (Li et al., 2006). For example, Jiang et al. (2010a, 2010b) and Zhou et al. (2007) analyzed B. purpurascens in Yunnan Province and found there are abundant morphological variations among different populations, and the content of the a.i.

Received for publication 14 Dec. 2020. Accepted for publication 15 June 2021.

Published online 20 July 2021

This study was funded by the Shanghai Seed Industry Development ProjectCollection of Characteristic Vegetable Resources, Selection of Characteristic Valuable Cultivars, and Standardization Breeding (Shanghai Agricultural Science Seeds (2015) No. 8), and Shanghai Chongming District Sustainable Development Science and Technology Innovation Action Plan-Integration and Application of Bergenia Planting Technology (CK2017-50).

We thank all the members who contributed to the collection of Bergenia resources. X.L. and Y.G. contributed equally to this work

Z.Y. is the corresponding author. E-mail: 347318085@qq.com.

This is an open access article distributed under the CC BY-NC-ND license (https://creativecommons.org/licenses/by-nc-nd/4.0/). (mainly bergenin and arbutin) is also significantly different. Wang et al. (2012) found rich genetic diversity and a high degree of genetic differentiation in $18 \mathrm{~B}$. purpurascens populations from different regions of Yunnan Province. However, the pedigree and genetic relationship of different Bergenia species are still unclear.

Intersimple sequence repeat (ISSR) is a dominant molecular marker first used by Zietkiewicz et al. (1994). It has many advantages, such as simple operation, low cost, sensitive, high polymorphism, small amount of DNA required, and no need to predict the genome sequence of the research object, and also the stability of simple sequence repeat. It is widely used to determine hybridity to evaluate genetic diversity (Andrea et al., 1998; Denduangboripant et al., 2010; Etminan et al., 2018; Yousefi et al., 2015; Zafar-Pashanezhad et al., 2020). The objective of our study was to explore the genetic relationships among Bergenia collected from the main distribution regions in China, and to provide a theoretical basis for wild resource conservation and their value for breeding and development.

\section{Materials and Methods}

Based on published literature and information provided by local forestry authorities, 28 Bergenia accessions containing six 
species and five uncertain accessions were collected. The six species were B. scopulosa (Fig. 1A), B. crassifolia (Fig. 1B), B. tianquaninsis (Fig. 1C), B. emeiensis (Fig. 1D-F), B. stracheyi (Fig. 1G), and B. purpurascens (Fig. 1H and I). Codes and origin information for the 28 accessions are shown in Table 1.

DNA EXtraction. About $100 \mathrm{mg} /$ plant of leaf tissue was used to extract genomic DNA using a modified hexadecyltrimethylammonium bromide (CTAB) procedure (Doyle and Doyle, 1987). Integrity and quality of DNA were evaluated by electrophoresis on $1.0 \%$ agarose gels. Concentrations of DNA were determined with an ultraviolet spectrophotometer (UV751GD; Jiang dong Precision Instrument Co., Suzhou, China).

ISSR PRIMER. ISSR primers were synthesized according to the primer set published by the University of British Columbia (Vancouver, BC, Canada). A total of 100 ISSR primers were synthesized, and all primers were tested using four Bergenia accessions: B. scopulosa (QL1), B. tianquaninsis (TQ1), B. emeiensis (EMHY), and B. purpurascens (BM1). Twenty-four primers with a strong amplified signal and good reproducibility were selected, and all 28 Bergenia accessions were evaluated with all 24 primers, for a total of 672 primer combinations. To confirm the reproducibility of the primers, more than two replicates were performed in the selection of the primers, and the repeatable bands were checked further when testing the Bergenia samples. The primer sequences are shown in Table 2.

Polymerase chain Reaction amplification. Polymerase chain reactions (PCRs) were performed in a $20-\mu \mathrm{L}$ reaction volume including $50 \mathrm{ng}$ genomic DNA, $1 \mu \mathrm{M}$ primer, $200 \mu \mathrm{M}$ deoxyribonucleotide triphosphates, $2 \mathrm{~mm} \mathrm{MgCl}_{2}, 1 \times$ Taq buffer, and $1 \mathrm{U}$ Taq DNA polymerase. The amplifications were performed with the following program: initial denaturation at $95^{\circ} \mathrm{C}$ for $5 \mathrm{~min}, 38$ cycles of $94^{\circ} \mathrm{C}$ for $1 \mathrm{~min}$, appropriate annealing temperature (see Table 2 for details) for $1 \mathrm{~min}$, and $72^{\circ} \mathrm{C}$ for 2 $\min$. The last extension was at $72^{\circ} \mathrm{C}$ for $10 \mathrm{~min}$. The PCR products were detected by $1.5 \%$ agarose gels, and a gel imaging system (model 2500; Tanon Technology Co., Shanghai, China) was used to generate images.

Statistical analysis. Popgene 32 software (Wang et al., 2010) was used to calculate various genetic diversity parameters: number of alleles, number of effective alleles $(\mathrm{Ne})$, the percentage of polymorphic bands, Shannon's information index $(I)$, and Nei's genetic diversity index $(H)$. The Dice similarity matrix (Nei and Li, 1979) was created from the genetic similarity coefficient using the SIMQUAL program of NTSYSpc 2.10 software (Rohlf, 2000). A dendrogram was drawn from the similarity matrices using the unweighted pair group method with arithmetic mean (UPGMA) following the SAHN function of NTSYSpc 2.10.

\section{Results}

AMPlifiCATION RESUlts AND POLYMORPHISM. Twenty-four primers producing clear bands, a strong signal, and good reproducibility were selected from 100 ISSR primers for PCR amplification of the 28 accessions (Table 2). The electrophoretograms amplified by primers 880,854 , and 876 are shown in Fig. 2. The amplified bands ranged from 200 to $3000 \mathrm{bp}$ and were mostly concentrated between 200 and $500 \mathrm{bp}$.

A total of 318 DNA bands were amplified by 24 primers from 28 DNA samples of Bergenia, of which 307 were polymorphic, with an average polymorphic rate of $96.54 \%$. The polymorphic rate of the 24 primers was more than $75 \%$, indicating there was a great genetic difference in the accessions of Bergenia. The number of bands amplified by the 24 primers was
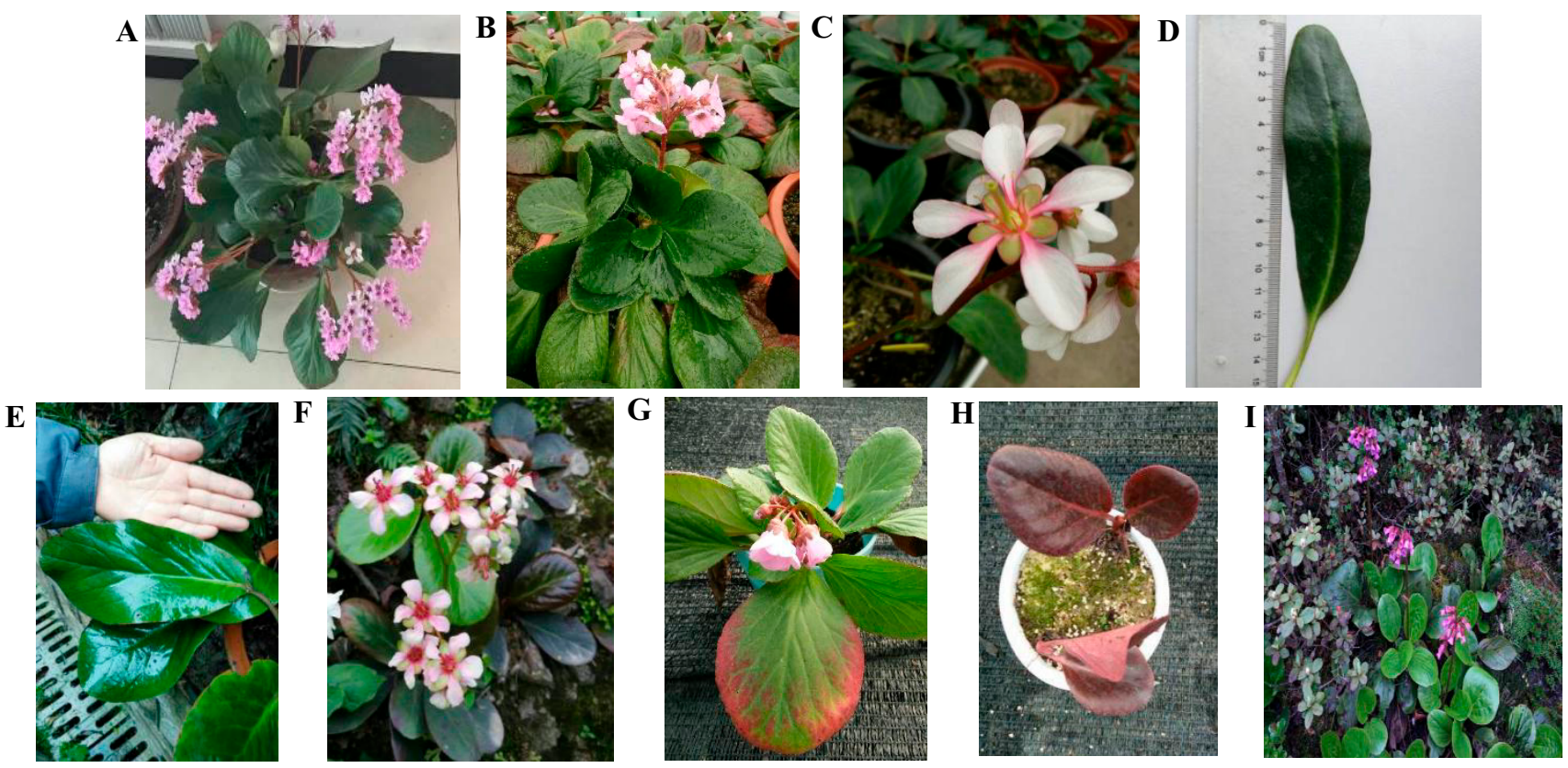

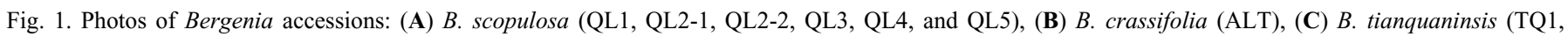
TQ2, TQ3, TQ4, and EM2-1), (D-F) B. emeiensis (EMHY, EM2-2, EM2-3, EM1-1, and EM1-2), (G) B. stracheyi (DB1 and DB2), (H, I) B. purpurascens (BM1, BM2, BM3, and BM4). QL1, QL2-1, QL2-2, QL3, QL4, and QL5 are the six accessions of $B$. scopulosa from different sites in Shaanxi Province. ALT is the accession from Altay, Xinjiang, autonomous region. TQ1, TQ2, TQ3, TQ4, and EM2-1 are the five accessions of B. tianquaninsis from different sites in Sichuan Province. EMHY, EM2-2, EM2-3, EM1-1, and EM1-2 are the five accessions of $B$. emeiensis from different sites in Sichuan Province. DB1 and DB2 are the two accessions of B. stracheyi from Nyingchi, Tibet, autonomous region. BM1, BM2, BM3, and BM4 are the four accessions of B. purpurascens from Bomi, Tibet Autonomous Region. 
Table 1. The 28 Bergenia accessions surveyed in China and their characteristics, origin, and geographic parameters.

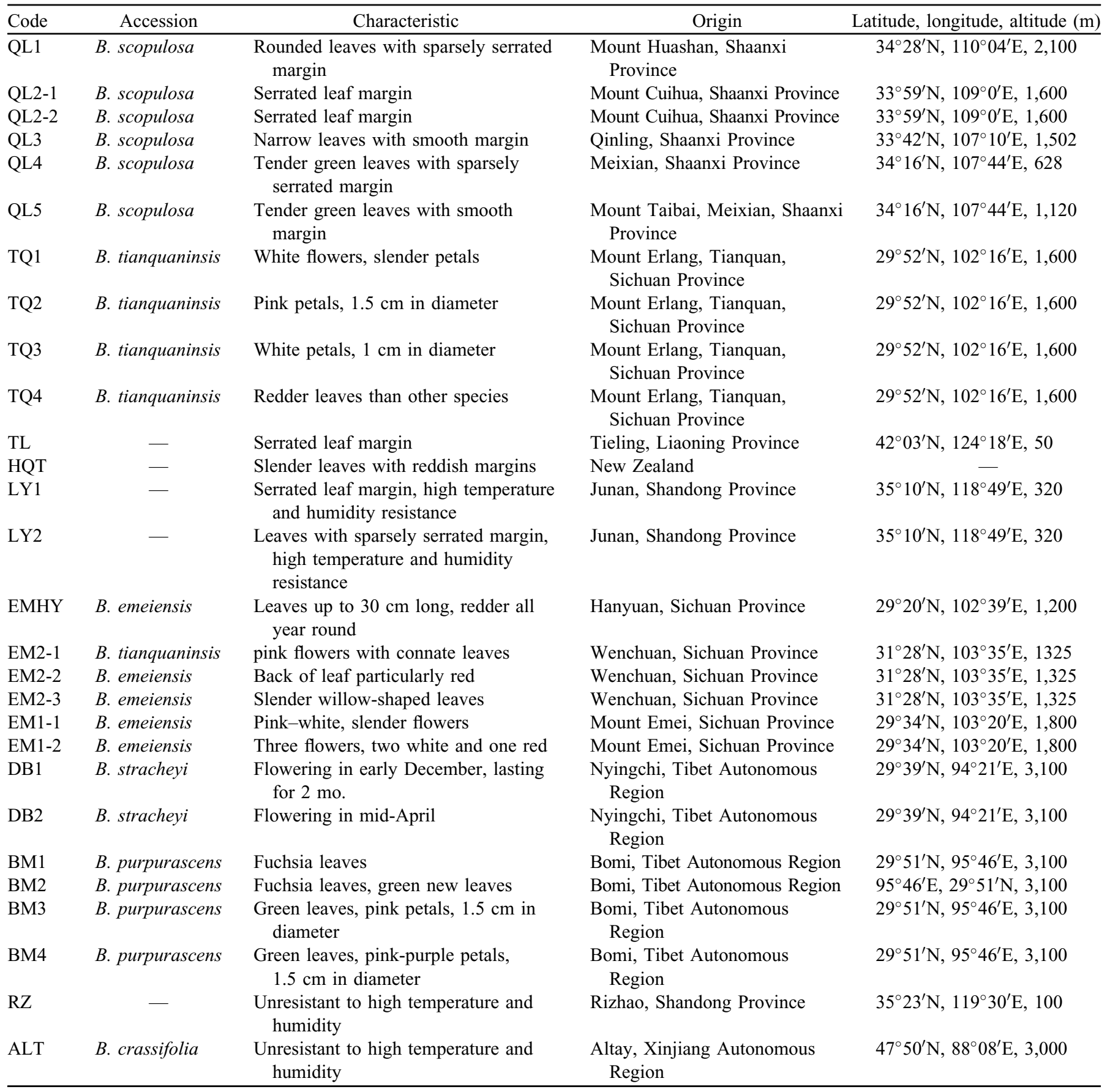

different, and an average of 13.25 bands were amplified per primer. The number of bands amplified by primer 881 was the largest $(n=23)$, whereas primer 890 amplified the least $(n=4)$. Moreover, the polymorphic percentage of the ISSR bands amplified by 24 primers was also different. Primer 890 showed the lowest rate $(75 \%)$ whereas the 14 primers showed the highest percentages $(100 \%)$ (Table 2).

Genetic DIVERsity. The number of effective alleles of the 28 Bergenia accessions ranged from 1.1217 to 1.6220 , and the average value was 1.3875 (Table 3 ). The maximum, minimum, and average values of $H$ were $0.4078,0.1022$, and 0.2455 , respectively. The corresponding values of $I$ were $0.5303,0.1937$, and
0.3805 , respectively. Our results indicate there were abundant genetic variations among the 28 accessions.

Cluster Analysis. The genetic similarity coefficient of the 28 accessions ranged from 0.4372 to 0.9765 , with an average of 0.5767. The coefficient between most accessions was less than 0.55 , indicating a large genetic difference among populations. Among them, the coefficient between QL2-1 and QL2-2, LY1, and LY2 was the largest, all of which were greater than 0.93 , indicating they were closely related. The coefficient between QL5 and BM1 was the smallest (0.4372), showing the greatest genetic difference.

Based on the genetic similarity coefficient matrices of the 28 accessions, the dendrogram was obtained using the UPGMA 
Table 2. Nucleotide sequences of 24 primers and their amplification results of 28 Bergenia accessions.

\begin{tabular}{|c|c|c|c|c|c|}
\hline Primer & Sequence of primer $\left(5^{\prime}-3^{\prime}\right)$ & $\begin{array}{l}\text { Annealing } \\
\text { temp }\left({ }^{\circ} \mathrm{C}\right)\end{array}$ & $\begin{array}{c}\text { Amplified } \\
\text { bands (no.) }\end{array}$ & $\begin{array}{c}\text { Polymorphic } \\
\text { bands (no.) }\end{array}$ & $\begin{array}{c}\text { Polymorphic } \\
\text { bands }(\%)^{\mathrm{z}}\end{array}$ \\
\hline UBC807 & AGAGAGAGAGAGAGAGT & 48 & 10 & 9 & 90 \\
\hline UBC810 & GAGAGAGAGAGAGAGAT & 48 & 10 & 8 & 80 \\
\hline UBC824 & ТСТСТСТСТСТСТСТСG & 52 & 10 & 10 & 100 \\
\hline UBC825 & ACACACACACACACACT & 54 & 8 & 8 & 100 \\
\hline UBC842 & GAGAGAGAGAGAGAGAYG & 52 & 11 & 11 & 100 \\
\hline UBC851 & GTGTGTGTGTGTGTGTYG & 48 & 12 & 12 & 100 \\
\hline UBC852 & TCTCTСТСТСТСТСТСRA & 51 & 14 & 14 & 100 \\
\hline UBC853 & TCTCTCTCTCTCTCTCRT & 51 & 12 & 11 & 91.67 \\
\hline UBC854 & TCTCTCTCTCTCTCTCRG & 53 & 20 & 20 & 100 \\
\hline UBC864 & ATGATGATGATGATGATG & 47 & 13 & 12 & 92.31 \\
\hline UBC874 & СССТСССТСССТСССТ & 63 & 15 & 15 & 100 \\
\hline UBC889 & DBDACACACACACACAC & 52 & 9 & 8 & 88.89 \\
\hline UBC890 & VHVGTGTGTGTGTGTGT & 48 & 4 & 3 & 75 \\
\hline UBC891 & HVHTGTGTGTGTGTGTG & 48 & 12 & 11 & 91.67 \\
\hline UBC892 & HVHTGTGTGTGTGTGTG & 50 & 19 & 19 & 100 \\
\hline UBC895 & AGAGTTGGTAGCTCTTGATC & 51 & 11 & 10 & 90.91 \\
\hline UBC899 & CATGGTGTTGGTCATTGTTCCA & 53 & 13 & 13 & 100 \\
\hline UBC900 & ACTTCCCCACAGGTTAACACA & 57 & 20 & 20 & 100 \\
\hline Total & - & - & 318 & 307 & - \\
\hline Avg & - & - & 13.25 & 12.79 & 96.54 \\
\hline
\end{tabular}

${ }^{\mathrm{z}}$ Polymorphic bands (no.)/amplified bands (no.).

method (Fig. 3). The 28 accessions were divided into three categories at the genetic similarity coefficient of 0.5475 . The first category includes B. scopulosa (QL1, QL2-1, QL2-2, QL3, QL4, and QL5) collected from Qinling Mountain, Shaanxi Province; potted seedlings collected from Tieling City, Liaoning Province (TL); and Junan County, Shandong Province (LY1 and LY2); as well as the cultivar HQT introduced from New Zealand. These accessions had straight and shaped leaves, with slightly shorter petioles, and they were better adapted to the high temperatures and humidity of Shanghai. Among them, QL1, QL3, QL4, and QL5 were all from Qinling Mountain, and they were clustered in a small group. The altitudes of QL4 and QL5 were 628 and $1120 \mathrm{~m}$, respectively; QL3 was $1502 \mathrm{~m}$, and QL1 had the highest altitude $(2100 \mathrm{~m})$. QL2-1 and QL2-2 were collected from Qinling Mountain at 1600 $\mathrm{m}$, and the genetic similarity between them was 0.9765 . Although the two accessions were less than $1 \mathrm{~km}$ apart geographically, they were still slightly different at the molecular level. LY1 and LY2 were collected from agricultural households $30 \mathrm{~km}$ away from Junan County, Shandong Province, and were clustered in a small group with high similarity. LY1 has been potted for more than 30 years, and the margin of its leaves is serrated. LY1 and LY2 showed the strongest adaptability with accession TL from Tieling City, Liaoning Province. They can survive the hot and humid summer in Shanghai with proper shade. The similarity of the three accessions was high. The cultivar HQT, introduced from New Zealand, was clustered in a single group, and its leaves are red and more slender than other accessions.

B. tianquaninsis (TQ1, TQ2, TQ3, TQ4, and EM2-1), B. emeiensis (EMHY, EM2-2, EM2-3, EM1-1, and EM1-2), $B$. stracheyi (DB1 and DB2), B. crassifolia [the accession from Altay, Xinjiang, autonomous region (ALT)], and the accessions collected from Rizhao City, Shandong Province (RZ), were clustered in the second category. The 12-year-old potted seedlings of RZ collected from Rizhao City, with a crown of $50 \mathrm{~cm}$, were clustered with B. crassifolia (Fig. 1B) collected from Altay, Xinjiang Uygur Autonomous Region, with a genetic similarity of 0.8142 . Both were resistant to cold but not to high temperatures and humidity, and their appearance was similar. Four Tianquan accessions (Fig. 1C)-TQ1, TQ2, TQ3, and TQ4 collected from the same location on Erlang Mountain, Sichuan Province, were clustered in a group. EM2-1, EM2-2, and EM2-3, collected from the same location on Mount Emei, were clustered in a group, of which EM2-3 was unique, with slender willow-shaped leaves (Fig. 1D), which was significantly different from the other two accessions. The genetic distance in the cluster analysis was also relatively long. EMHY, collected from Hanyuan County, Sichuan Province, has a leaf length of $30 \mathrm{~cm}$, a petiole of less than $5 \mathrm{~cm}$, and thick leaves (Fig. 1E). It was different from other Emei accessions in appearance (Fig. 1F) and it was clustered in a single group. In addition, EM1-1 and EM1-2 collected from Mount Emei, Sichuan Province, were clustered with DB1 and DB2 collected from Nyingchi County, Tibet Autonomous Region (Fig. 1G).

The accessions from Bomi County, Tibet Autonomous Region (BM1, BM2, BM3, and BM4), were clustered separately in the third category. Under the same cultivation conditions, BM1 and BM2 (Fig. 1H), with purple leaves, were clustered together, whereas BM3 and BM4 (Fig. 1I), with green leaves, 
A
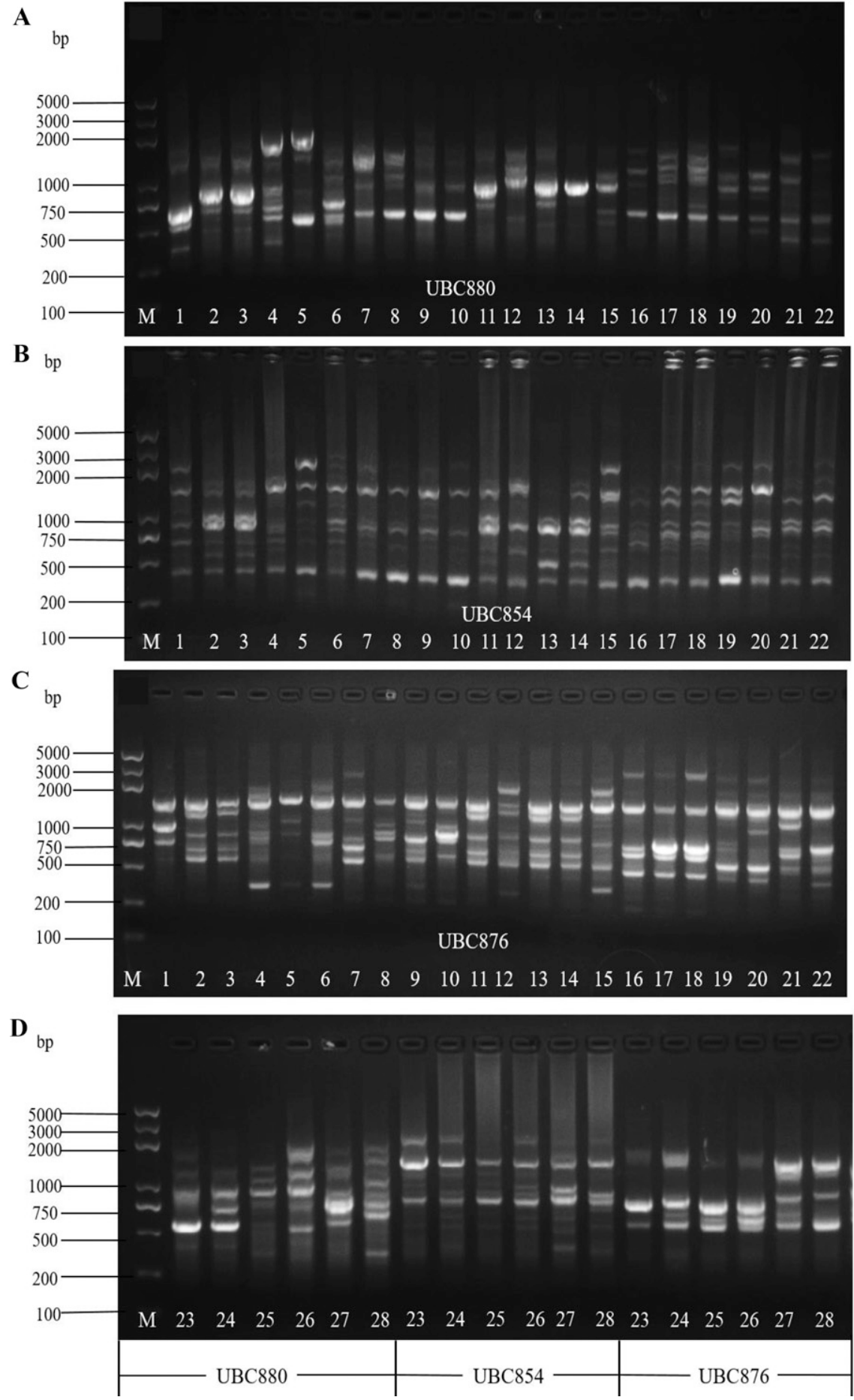

Fig. 2. The intersimple sequence repeat polymerase chain reaction amplification results of the 28 Bergenia accessions with primers of (A) UBC880, (B) UBC854, and (C) UBC876. M refers to DNA marker. The standard molecular weight is shown on the left. The 28 Bergenia accessions are B. scopulosa (nos. 1-6); B. tianquaninsis (nos. 7-10); TL, which is the accession from Tieling, Liaoning Province; HQT, which is the accession introduced from New Zealand; LY1 and LY2, which are the accessions with different leaf characteristics from Junan, Shandong Province; $B$. emeiensis (no. 15); B. tianquaninsis; B. emeiensis (nos. 17-20); B. stracheyi (nos. 21 and 22); $B$. purpurascens (nos. 23-26); RZ, which is the accession from Rizhao, Shandong Province; and $B$. crassifolia. Because each gel plate can only hold 22 samples, samples 23 through 28 of each primer are nested in (D).

were clustered in a group. This finding indicates that leaf color of Bergenia accessions from the same origin is different, and molecular identification was also divided into two groups.

\section{Discussion}

In our study, the ISSR technique was used to assess genetic diversity of Bergenia taxa collected from China, and a suitable reaction system for ISSR-PCR analysis of this genus was established. The optimized system was used to screen the polymorphic primers to ensure clear and accurate amplification results. Our results indicate that the ISSR marker had high polymorphism in the 28 Bergenia accessions, which reflected the genetic relationship among multiple origins accurately, and was suitable for assessing the genetic diversity of the genus.

There are 10 species of Bergenia in the world; seven of them are distributed in China. Wang et al. (2012) analyzed 18 accessions of B. purpurascens across Yunnan Province by ISSR molecular markers. They found that the Dice similarity coefficient between different accessions ranged from 0.651 to 0.841 , and the accessions collected from the same origin were not clustered together completely, indicating that different accessions within the same species had a high degree of genetic differentiation and rich genetic diversity. In our study, the 28 accessions collected from six provinces were amplified by 24 primers, and 318 bands were amplified, of which 307 were polymorphic bands, with a polymorphism rate of $95.01 \%$. Moreover, the similarity coefficient ranged from 0.4372 to 0.9765 ; and the values of $N_{e}, H$, and $I$ were 1.3875, 0.2455 , and 0.3805 , respectively, which indicates that the collected Bergenia germplasm have extremely rich genetic diversity at the molecular level. This is because there is almost no genetic exchange between different accessions. Wild Bergenia grows in the inaccessible alpine zone; the excavated germplasm is consumed as medicinal materials; and there is currently no artificial cultivation, breeding, and hybridization.

There have been many reports on the relationship among plant germplasm based on ISSR molecular markers. Li et al. (2011) assessed the genetic relationship between Medicago ruthenica germplasm in Inner Mongolia and found that germplasm with a close geographic distance first clustered together, but Du et al. (2009) found that the germplasm clustering was not related completely to geographic distribution when studying Brassica rapa ssp. pekinensis germplasm. Based on the ISSR molecular markers, we performed cluster analysis on 28 Bergenia accessions and found cases when the same species from the same region were clustered together completely, such as the four
B. tianquaninsis (TQ1, TQ2, TQ3, and TQ4), the two Bergenia (LY1 and LY2), and the four B. purpurascens (BM1, BM2, BM3, 
Table 3. Genetic diversity indices of 28 Bergenia accessions. The maximum, minimum, and mean values of $N_{\mathrm{e}}, H$, and $I$ are in bold type.

\begin{tabular}{|c|c|c|c|c|}
\hline Primer & $N_{a}$ & $N_{e}$ & $H$ & $I$ \\
\hline UBC807 & 2 & 1.5626 & 0.3422 & 0.5168 \\
\hline UBC810 & 1.9 & 1.4301 & 0.2564 & 0.3905 \\
\hline UBC815 & 1.875 & 1.5309 & 0.2917 & 0.4282 \\
\hline UBC824 & 2 & 1.6220 & 0.3584 & 0.5303 \\
\hline UBC 825 & 1.875 & 1.5231 & 0.3003 & 0.4456 \\
\hline UBC842 & 2 & 1.5107 & 0.2958 & 0.4496 \\
\hline UBC845 & 1.9091 & 1.5610 & 0.4078 & 0.4663 \\
\hline UBC851 & 2 & 1.5091 & 0.3136 & 0.4832 \\
\hline UBC852 & 2 & 1.4576 & 0.2825 & 0.4391 \\
\hline UBC853 & 1.9167 & 1.5262 & 0.3081 & 0.4603 \\
\hline UBC854 & 2 & 1.4331 & 0.2774 & 0.4368 \\
\hline UBC864 & 1.9231 & 1.3990 & 0.2324 & 0.3582 \\
\hline UBC874 & 1.9333 & 1.3089 & 0.2113 & 0.3478 \\
\hline UBC876 & 1.9444 & 1.3574 & 0.2373 & 0.3814 \\
\hline UBC878 & 2 & 1.2854 & 0.1860 & 0.3107 \\
\hline UBC 880 & 2 & 1.3316 & 0.2202 & 0.3608 \\
\hline UBC881 & 2 & 1.3985 & 0.2473 & 0.3908 \\
\hline UBC889 & 1.8889 & 1.4078 & 0.2502 & 0.3909 \\
\hline UBC890 & 1.75 & 1.1217 & 0.1022 & 0.1937 \\
\hline UBC891 & 1.9167 & 1.2332 & 0.1754 & 0.3016 \\
\hline UBC892 & 2 & 1.2732 & 0.1779 & 0.2998 \\
\hline UBC895 & 1.9091 & 1.1609 & 0.1226 & 0.2228 \\
\hline UBC899 & 2 & 1.1476 & 0.119 & 0.2241 \\
\hline UBC 900 & 2 & 1.2092 & 0.1768 & 0.3017 \\
\hline Mean & 1.9476 & 1.3875 & 0.2455 & 0.3805 \\
\hline $\mathrm{SD}$ & 0.1909 & 0.3257 & 0.1625 & 0.2133 \\
\hline
\end{tabular}

$N_{a}=$ number of alleles; $N_{e}=$ number of effective alleles; $H=$ Nei's genetic diversity index; $I=$ Shannon's information index.

and BM4). However, there were also cases when the same species from the same region did not cluster together completely, such as the six B. scopulosa (QL1, QL2-1, QL2-2, QL3, QL4, and QL5) and the five B. emeiensis (EMHY, EM1-1, EM1-2, EM2-2, and

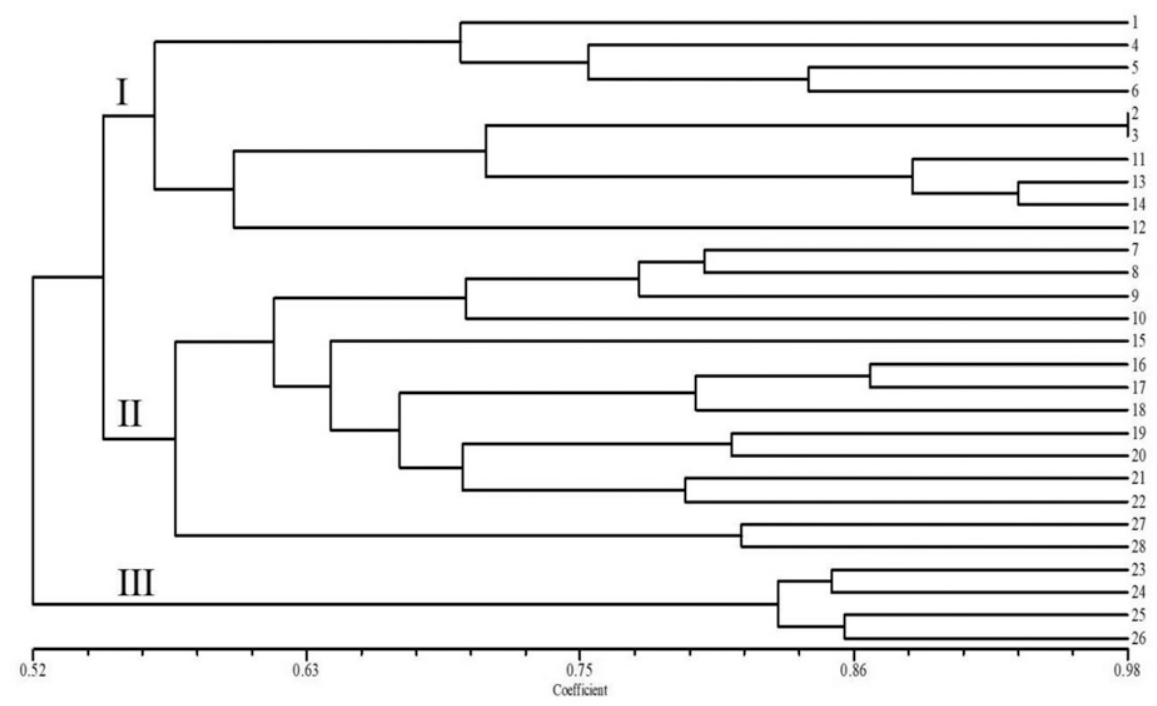

Fig. 3. Depictions of the unweighted pair group method with arithmetic mean clustering analysis of the 28 Bergenia accessions. The 28 Bergenia accessions are B. scopulosa (nos. 1-6); B. tianquaninsis (nos. 7-10); TL, which is the accession from Tieling, Liaoning Province; HQT, which is the accession introduced from New Zealand; LY1 and LY2, which are the accessions with different leaf characteristics from Junan, Shandong Province; B. emeiensis (no. 15); B. tianquaninsis; B. emeiensis (nos. 17-20); B. stracheyi (nos. 21 and 22); B. purpurascens (nos. 23-26); RZ, which is the accession from Rizhao, Shandong Province; and B. crassifolia.
EM2-3). The reason for this phenomenon may be the result of specific microhabitats, leading to obvious genetic differentiation between different accessions. During the investigation, we found that although some accessions were collected from the same region (such as the six B. scopulosa accessions from the Qinling Mountain, Shaanxi Province), their specific microhabitats (e.g., soil condition, slope, aspect, light, altitude, associated plants) were different, and their morphological characteristics were also significantly different. Some accessions showed rounded leaves, some showed narrower leaves, and some had serrated leaf margins. Currently, there are relatively few cultivars of Bergenia (Jiang, 2010; Zhou et al., 2007). The ISSR identification results indicate there is a huge space for breeding new cultivars in the future.

The unknown accession RZ showed the same adaptability as $B$. crassifolia (ALT), and the genetic similarity between the two was greater than 0.8 , which was determined as $B$. crassifolia. The age of potted seedlings TL, LY1, and LY2 has exceeded 10 years, but the origin of these accessions cannot be verified. Through ISSR molecular identification, they clustered together with species B. scopulosa, and were judged as B. scopulosa. These accessions can survive the high temperatures and humidity of Shanghai in the summer, showing strong adaptability. However, the ecological habits and cultivation techniques of these accessions need to be explored further. Such accessions can be developed in the future and promoted throughout the Yangtze River Delta and even across China.

The BM1 (Fig. 1H) collected from Bomi County, Tibet Autonomous Region, was extremely cold resistant, and its flowers were fuchsia. After potting in Shanghai, the flowers and leaves were fuchsia, and it is expected to develop into colored groundcover, but BM1 cannot survive the high temperatures and humidity of Shanghai in the summer. B. scopulosa showed strong adaptability and can survive the summer easily. Because these two accessions are the most distantly related among the collected germplasm, they can be used as breeding parents to cultivate fuchsia accessions that can survive the summer climate. In addition, B. crassifolia had the highest content of bergenin among all accessions (Wang et al., 2006), and it was clustered in different categories with $B$. scopulosa and B. purpurascens. The genetic similarity coefficients of the three accessions were $0.5244,0.5586$, and 0.4372 , respectively. Their selection and application will not only increase the genetic diversity of the genus, but also will complement each other for different purposes. The UPGMA dendrogram based on ISSR molecular markers showed the genetic relationship of the 28 accessions at the molecular level, which provides a theoretical basis for the promotion and application of valuable accessions, and the selection of breeding parents, and thus can effectively avoid the blindness of valuable accession selection and simplification of the genetic basis.

\section{Literature Cited}

Andrea, D.W., Q.Y. Xiang, and S.R. Kephart. 1998. Assessing hybridization in natural population of Penstemon (Scrophulariaceae) using hypervariable inter-simple sequence repeat 
(ISSR) bands. Mol. Eco1. 7(9):1107-1125, doi: 10.1046/ j.1365-294x.1998.00425.x.

Denduangboripant, J., S. Setaphan, W. Suwanprasart, and S. Panha. 2010. Determination of local tobacco cultivars using ISSR molecular marker. Chiang Mai J. Sci. 37(2):293-303.

Doyle, J.J. and J.L. Doyle. 1987. A rapid DNA isolation procedure for small quantities of fresh leaf tissue. Phytochem. Bull. 19:11-15.

Du, L.M., T.H. Hu, C.L. Bao, Q.M. Zhu, H.J. Hu, and W.H. Mao. 2009. Genetic diversity and genetic relative's analysis of pakchoi germplasm based on inter-simple sequence repeat (ISSR). Chin. Agr. Sci. Bull. 25(11):20-24 (in Chinese with English abstract).

Etminan, A., A. Pour-Aboughadareh, A. Noori, A. Ahmadi-Rad, L. Shooshtari, Z. Mahdavian, and M. Yousefiazar-Khanian. 2018. Genetic relationships and diversity among wild salvia accessions revealed by ISSR and SCoT markers. Biotechnol. Biotec. Eq. 32(3):610-617, doi: 10.1080/13102818.2018.1447397.

Jiang, H.J. 2010. Investigation and evaluation of Bergenia purpurascens (Hook. f. et Thoms.) Engl. resources in Yunnan Province. Yunnan Agricultural University, Kunming, China, PhD Diss.

Jiang, H.J., F.G. Guo, L.M. Zhang, Y.P. Chen, S.W. Li, and F. Yang. 2010a. Comparison of arbutin contents from Bergenia purpurascens in Yunnan. Chin. J. Chin. Mater. Med. 35(14):1812-1814, doi: 10.4268/cjcmm20101408.

Jiang, H.J., F.G. Guo, L.M. Zhang, Y.P. Chen, and S.C. Yang. 2010b. Comparison of bergenin contents of Bergenia purpurascens among different regions in Yunnan Province. J. Yunnan Agr. Univ. 25(6):895898, doi: 10.3969/j.issn.1004-390X.2010.06.027.

Li, H.Y., Z.Y. Li, X.L. Wang, W.G. Shi, and J.J. Xing. 2011. Genetic diversity studies of Medicago ruthenica in Inner Mongolia by ISSR. Acta Bot. Boreali-Occident. Sin. 31(1):52-56.

Li, W.C., F.G. Guo, L.M. Zhang, H.M. Yu, X. Li, and C. Lin. 2006. The situation and prospect of research on Bergenia purpurascens. J.
Yunnan Agr. Univ. 21(6):845-850, doi: 10.3969/j.issn.1004-390X. 2006.06.031.

Nei, M. and W.H. Li. 1979. Mathematical model for studying genetic variation in terms of restriction endonucleases. Proc. Natl. Acad. Sci. USA 76:5269-5273, doi: 10.1073/pnas.76.10.5269.

Rohlf, F.J. 2000. NTSYS-pc: numerical taxonomy and multivariate analysis system. Version 2.1. Exeter Software, New York, NY.

Wang, J.L., J. He, C. Zou, and Q.W. Li. 2006. Research advancement of bergenin. Chin. J. Ethnomed. Ethnopharm. 83(6):321-325, doi: 10.3969/j.issn.1007-8517.2006.06.007.

Wang, S.Y., F.G. Guo, Y.H. Zhang, and Z.A. Yang. 2012. ISSR analysis of 18 Bergenia purpurascens resources in Yunnan. Chin. Agr. Sci. Bull. 28(7):114-118, doi: 10.3969/j.issn.1000-6850.2012.07.022.

Wang, Y.Q., Y. Fu, Q. Yang, N. Luo, Q.X. Deng, J. Yan, J.G. Zeng, and G.L. Ruan. 2010. Genetic diversity of Eriobotrya analyzed by ISSR markers. Sci. Silv. Sin. 46(4):49-57, doi: 10.11707/j.1001-7488. 20100408.

Yousefi, V., A. Najaphy, A. Zebarjadi, and H. Safari. 2015. Molecular characterization of Thymus species using ISSR markers. J. Anim. Plant Sci. 25(4):1087-1094.

Zafar-Pashanezhad, M., E. Shahbazi, P. Golkar, and B. Shiran. 2020. Genetic variation of Eruca sativa L. genotypes revealed by agromorphological traits and ISSR molecular markers. Ind. Crops Prod. 145:111992, doi: 10.1016/j.indcrop.2019.111992.

Zhou, G.Y., W.C. Li, and F.G. Guo. 2007. Resource investigation and observation of biological characteristics of Bergenia purpurascens (Hook. f. et Thoms.) Engl. Chin. Agr. Sci. Bull. 23(5):390-392, doi: 10.3969/j.issn.1000-6850.2007.05.089.

Zietkiewicz, E., A. Rafalski, and D. Labuda. 1994. Genome fingerprinting by simple sequence repeat (SSR)-anchored polymerase chain reaction amplification. Genome 20:176-183, doi: 10.1006/geno. 1994.1151. 